\title{
LUTTE INTEGREE CONTRE LA MALADIE DES RAIES NOIRES (MRN) DES BANANIERS PLANTAIN (Musa, AAB, cv Orishele) SOUS DIFFERENTES DENSITES DE PLANTATION
}

\author{
K.KOBENAN'1, S. TRAORE' ${ }^{2}$, G.P.GNONHOURI' et N. T. YAO ${ }^{3}$ \\ ${ }^{1}$ Laboratoire de défense des cultures, Centre National de Recherche Agronomique (CNRA), \\ Station de recherche de Bimbresso, 01 BP 1536 Abidjan 01 Côte d'Ivoire \\ 2Laboratoire de Physiologie Végétale, UFR Biosciences Université de Cocody, 22 BP 582 Abidjan 22 \\ 'Laboratoire d'agro- pédologie, Centre National de Recherche Agronomique (CNRA), \\ station de recherche de Bimbresso, 01 BP 1536 Abidjan 01 Côte d'Ivoire
}

\begin{abstract}
RESUME
L'étude a été conduite à la station expérimentale du CNRA à Azaguié, au Sud de la Côte d'lvoire $\left(4^{\circ} 05^{\prime} \mathrm{W} ; 5^{\circ} 38^{\prime} \mathrm{N}\right)$. Elle a porté sur l'incidence de la densité de plantation et du traitement fongique sur la cercosporiose en culture de bananier plantain. Les effets de 3 densités de plantation (1667, 2500 et 3333 plants/ha), avec 2 niveaux de contrôle de la maladie des raies noires (MRN) (traité et non traité) dans un dispositif en blocs de Fisher, à 4 répétitions, ont été comparés. De la plantation au $7^{e}$ mois, le contrôle de la MRN a été assuré uniquement par la suppression des parties nécrosées du limbe foliaire, toutes les 2 semaines. A partir du $8^{e}$ mois, des applications mensuelles du fongicide trifloxystrobine (à $100 \mathrm{~g}$ de m.a ; en émulsion dans l'huile banole à raison de $18 \mathrm{l} / \mathrm{ha}$ ) ont été assurées uniquement sur les parcelles traitées. Les résultats ont permis de mettre en évidence l'absence d'interaction entre la densité de plantation et le contrôle de la MRN. Le nombre de feuilles vivantes, la circonférence du faux tronc, les poids moyens du régime ( $10 \%$ en plus) et du doigt médian interne de la $4^{\mathrm{e}}$ main ont été plus élevés pour les parcelles traitées. Les applications du fongicide ont eu le meilleur effet sur la végétation pour une densité de 2500 plants /ha. Les améliorations de ce résultat sont discutées en rapport avec les perspectives d'amélioration des revenus des producteurs.
\end{abstract}

Mots clés : Bananier plantain, densité de plantation, maladie des raies noires, trifloxystrobine, lutte intégrée, Côte d'lvoire.

\begin{abstract}
INTEGRATED CONTROL OF BLACK SIGATOKA ( BLACK STREAKS DISEASE ; MRN) IN PLANTAIN (MUSa, AAB, CV Orishele) UNDERDIFFERENTPLANTING DENSITIES
\end{abstract}

The study conducted at the Azaguié Experiment Station of CNRA in Southern Côte d'Ivoire $\left(4^{\circ} 05^{\prime} \mathrm{W} ; 5^{\circ} 38^{\prime} \mathrm{N}\right)$ aims at assessing the effects of planting densities and fungicide applications on MRN in plantain. Three cultivation densities (1666, 2500 and 3333 plants/ha) and 2 levels of fungicide (treated and untreated) were tested in a Fisher bloc experiment with 4 replications. From planting to the $7^{\text {th }}$ month, MRN was controlled on all hole plots by physical elimination of injured parts of the foliar lamina. Monthly applications of fungicide trifloxystrobin $100 \mathrm{~g}$ of a.i., in banole oil ; $18 \mathrm{l} / \mathrm{ha}$ ) from the $8^{\text {th }}$ to the $11^{\text {th }}$ month, resulted in a complete control of the disease. Results showed that fungicide applications improved vegetation and increased yield by about $10 \%$. A density of 2500 plants/ha gave the best vegetation. Results obtained were discussed, with particular emphasis on the possible increase in the level of fungicide applications in relation with farmers income.

Key words : Plantain, planting density, black streak disease, trifloxystrobin integrated control, Côte d'lvoire. 


\section{INTRODUCTION}

En provoquant une sénescence prématurée des feuilles, la maladie des raies noires (MRN) à Mycosphaerella fijiensis des bananiers et des bananiers plantain occasionne souvent des pertes de rendements estimées entre 20 et $50 \%$ (BURT et al., 1997) selon les cultivars et les conditions d'expérimentation. La lutte chimique contre cette maladie est bien maîtrisée dans les unités industrielles de production de banane de bouche. Les applications systématiques de suspensions huileuses de fongicides, soit à l'aide de pulvérisateurs à dos motorisés, soit au moyen d'aéronefs sont courants dans les grandes unités de production de bananes. Dans certaines zones de production industrielle, la lutte chimique s'est déclenchée sur prévision à partir de l'observation des données météorologiques (Ganry et Meyer, 1972a et b ; Ganry et Laville, 1983). Cette prévision dite "d'avertissement climatique» permet une réduction du nombre annuel des traitements à 6 ou 8 contre 12 à 18, voire 24 habituellement. A l'inverse, dans les exploitations extensives de bananier plantain, la lutte contre cette maladie est quasi inexistante, en raison des surcoûts qu'elle occasionne et du manque de technicité. Cette situation entraine, en partie, les faibles rendements observés en milieu paysan. En effet, la MRN en réduisant la surface active de la feuille chez les variétés sensibles, entraîne un mauvais remplissage des fruits chez les variétés traditionnelles de bananiers plantain.

Les travaux de Belalcazar (2001) ont montré qu'en combinant la densité de plantation et la suppression systématique des parties nécrosées du limbe, il est possible de réduire l'incidence de la MRN sur le rendement, même en l'absence de toute application de fongicide.

Chez le bananier, au moins 8 feuilles saines au moment de la floraison sont indispensables à un remplissage correct des fruits (Lassoudière, 1978). Etant donné que l'émission foliaire s'arrête avec la jetée de l'inflorescence du bananier, l'équation à resoudre consiste à obtenir à la phase pré florale un nombre de feuilles saines supérieur ou égal à 8 et de les maintenir dans cet état pendant la période de remplissage du régime. Pour atteinte cet objectif, 2 paramètres ont été combinés : un programme minimum et tardif d'application de fongicide, et l'accroissement de la densité de plantation.

\section{MATERIEL ET METHODES}

\section{MATERIEL VEGETAL}

La variété Orishele de bananier plantain connue pour son bon potentiel de production et sa forte sensibilité à la maladie des raies noires a été utilisée. Le matériel de plantation a été constitué par des plants issus de culture in vivo produits à la station d'expérimentale du Centre National de Recherche Agronomique (CNRA) à Azaguié.

\section{SITE DE L'ETUDE}

L'étude a été conduite sur l'unité pilote du CNRA à Azaguié $(50 \mathrm{Km}$ au Nord-Est d'Abidjan, $80 \mathrm{~m}$ d'altitude, $04^{\circ} 05^{\prime} \mathrm{W}$ et $05^{\circ} 38^{\prime} \mathrm{N}$ ). La parcelle expérimentale est une jachère de 4 ans, dont la flore dominante est constituée par Chromolaena odorata. Le sol est du type ferralitique fortement désaturé, hydromorphe, à texture-argilo-sablo- limoneuse. Le régime pluviométrique est de type bimodal à 4 saisons : une grande (de mars à juin) et une petite (de septembre à novembre) saisons pluvieuses ; et une grande (de décembre à février) et une petite (de juillet à août) saisons sèches. Les températures moyennes varient entre 24 et $28^{\circ} \mathrm{C}$.

\section{DISPOSITIF EXPERIMENTAL}

Le dispositif utilisé est un bloc de Fisher à 4 répétitions, comportant 2 facteurs : la densité de plantation, avec 3 niveaux (D1: 1667 ; D2 : 2500 et D3 : 3333 plants/ha) et le contrôle de la maladie des raies noires avec 2 niveaux (parcelles recevant des applications de fongicides $(T)$ et sans application de fongicide (NT). Il y a eu au total 24 parcelles élémentaires de $300 \mathrm{~m}^{2}(15 \mathrm{~m} \times 20 \mathrm{~m})$.

\section{PLANTING}

Après fauchage, la biomasse de $C$. odorata a été abandonnée sur place pour servir de mulch aux bananiers du $1^{\text {er }}$ cycle cultural. Le mulch a été étalée dans les interlignes de bananiers plantain après planting. 
La trouaison a été réalisée manuellement avec des trous de dimensions $30 \times 30 \times 30 \mathrm{~cm}$ quelle que soit la densité. La mise en terre des vivo plants a eu lieu le 15 mai 2002. Une irrigation d'appoint de $4 \mathrm{~h}$, tous les $3 \mathrm{j}$, a été apportée pendant les mois de décembre à avril.

Après plantation, les parcelles ont été manuellement et régulièrement sarclées. Trois mois après planting, des traitements périodiques d'herbicides "gramoxone" ont permis de supprimer les mauvaises herbes afin de faciliter une bonne croissance des bananiers.

A la plantation, chaque plant a reçu $250 \mathrm{~g}$ de Dolomie et $200 \mathrm{~g}$ de phosphate tricalcique comme engrais de fond. Au cours du développement végétatif les apports d'engrais ont été effectués mensuellement, à raison de $40 \mathrm{~g}$ d'urée/plant et $50 \mathrm{~g}$ de chlorure de potasse par plant.

\section{TRAITEMENTS FONGIQUES}

Au $8^{\mathrm{e}}$ mois après la mise en terre des vivo plants, toutes les parcelles ont reçu le même traitement vis-à-vis de la cercosporiose noire : suppression physique systématique des parties nécrosées du limbe foliaire toutes les 2 semaines, quel que soit l'étage foliaire attaqué. Les parcelles traitées ont reçu en plus des ablations foliaires, des applications tardives de fongicides réalisées à $8,9,10$ et 11 mois après plantation. Pendant cette période d'application de fongicides la suppression systématique des parties nécrosées du limbe s'est poursuivie sur toutes les parcelles jusqu'à la veille des premières récoltes. Les applications de fongicides, ainsi que la gestion mécanique de la MRN ont été arrêtées après celles du $11^{\mathrm{e}}$ mois.

Le fongicide Tega, dont la matière active est (trifloxystrobine), appartient à la famille des strobilurines. Elle a été utilisée à la dose unique de $100 \mathrm{~g} \mathrm{~m}$.a. /ha. Les applications ont eu lieu dans une émulsion huileuse (Banole), à raison de $18 \mathrm{l} / \mathrm{h}$. Les traitements ont été effectués au moyen d'un pulvérisateur à dos motorisé (atomiseur) de marque «solo».

Sur chaque parcelle, les 10 bananiers plantain centraux ont été observés. Les relevés ont porté sur : au plan phytosanitaire : le nombre de feuilles vivantes (NFV); la Plus Jeune Feuille avec Tirets (PJFT, la feuille portant au moins 10 tirets individualisés, a été comptée à partir du cigare) ; la Plus Jeune Feuille Nécrosée (PJFN, la feuille portant au moins 10 nécroses individualisées) et au plan agronomique : l'intervalle floraison récolte (IFR, (j)); le poids moyen du doigt médian interne de la $4^{\mathrm{e}}$ main (PDR, $(\mathrm{g})$ ) et le poids moyen du régime $(P R,(k g))$.

\section{ANALYSES DES DONNEES}

Les données recueillies ont été soumises à une analyse de la variance à l'aide du logiciel STATITCF. La comparaison des moyennes des traitements a été faite à l'aide du test de Newman-Keuls, au seuil de $5 \%$.

\section{RESULTATS}

\section{ANALYSE STATISTIQUE}

L'analyse de la variance n'a pas montré d'interaction entre la densité de plantation et le contrôle de la cercosporiose noire. Pour cela, les comparaisons des moyennes ont été faites séparément pour les différents paramètres étudiés. De plus, seuls les paramètres en rapport direct avec l'état sanitaire des feuilles ont été pris en compte dans cette étude.

\section{Nombre de feuilles vivantes et intervalle plantation-floraison}

L'incidence de la maladie des raies noires sur le nombre de feuilles vivantes n'a pas significativement varié avec la densité de plantation pour les parcelles non traitées. Pour les parcelles ayant reçu le fongicide, ce nombre a varié significativement (Tableau 1A). En ce qui concerne l'intervalle de temps entre la plantation et la floraison (IPF), il n'y a pas eu de différence significative entre les densités de plantation comparées, quel que soit le mode de gestion de la cercosporiose (Tableau 1A). Toutefois, l'analyse traitement par traitement (Tableau 1B) montre certes des variations, mais elles n'ont pas été significatives. 
Tableau 1 : Nombre de feuilles vivantes et intervalle de temps entre la plantation et la floraison des bananiers en fonction des densités.

Number of active leaves and time to flowering of plantain trees at various planting densities.

\begin{tabular}{l}
\cline { 2 - 6 } \\
\cline { 2 - 7 }
\end{tabular}

\section{Circonférence du faux tronc}

La circonférence du faux tronc a significativement varié avec la densité de plantation, d'une part, et avec le traitement fongicide, d'autre part. Les densités élevées ont montré les plus faibles circonférences du faux tronc. Les parcelles ayant reçu des doses du fongicide ont montré les circonférences de faux tronc les plus élevées, quelle que soit la densité de plantation. Toutefois, l'effet du traitement fongicide a été plus marqué pour les densités D1 et D2 que pour la densité D3 (Figure 1).

\section{Etage foliaire attaqué}

Pendant la phase précédant les applications du fongicide, lorsque la gestion de la MRN était assurée uniquement par la suppression physique des parties nécrosées du limbe foliaire, une aggravation de la maladie a été observé avec la densité de plantation. Les courbes des rangs des plus jeunes feuilles attaquées (avec tirets ; PJFT) par la maladie ont été relativement stables (Figure 2). Suite aux applications du fongicide en janvier et en février, les courbes montrent une phase ascendante, accentuée surtout pour les parcelles traitées (Figure 2). Par la suite, à l'approche de la récolte, une chute a été observée.

En ce qui concerne la plus jeune feuille nécrosée (PJFN), les moyennes générales des parcelles traitées ont été significativement supérieures à celles des parcelles non traitées (Figure 3). Les applications fongicides ont été les plus efficaces avec la densité D2. Pour celle-ci, la différence entre parcelles traitées avec le fongicide et parcelles non traitées a atteint 3 feuilles en faveur des parcelles traitées. Cette différence a été inférieure à 2 feuilles pour la densité D3 et légèrement supérieure à 2 feuilles pour la densité D1 (Figure 3).

\section{Intervalle Floraison-Récolte}

L'intervalle entre la floraison et la récolte a été affectée, de façon significative, par la maladie. En effet, les bananiers non traités ont été récoltés 77 j contre 82 j après floraison pour les plants ayant reçu le programme minimum de lutte intégrée.

\section{Poids des fruits}

La maladie des raies noires a affecté significativement le poids des fruits. Les bananiers traités ont eu en moyenne des doigts de $274 \mathrm{~g}$. Ce poids n'a été que de $256 \mathrm{~g}$ en l'absence d'applications fongicides. Ce qui correspond à une différence de $18 \mathrm{~g}(8,38 \%)$ en faveur des parcelles traitées.

\section{Effet des densités de plantation}

Le poids des régimes a été réduit de façon notable par l'accroissement des densités. Le poids moyen des régimes a baissé avec la densité de plantation. Les fortes densités ont constitué un groupe homogène $(12,79 \mathrm{~kg}$ pour D2 et 11,35 kg pour D3), avec des poids de régimes significativement inférieurs à ceux de D1 $(15,25 \mathrm{Kg})$ et correspondant à des variations respectives de 19,23 et 34,36\% (Tableau 2).

\section{Effet de la cercosporiose}

Des différences significatives ont été observéés entre les poids des régimes sur les parcelles traitées $(13,87 \mathrm{~kg})$ contre $12,39 \mathrm{~kg}$ sur les parcelles non traitées correspondant à une variation de $12 \%$ en faveur des premières (Tableau 2 ). 


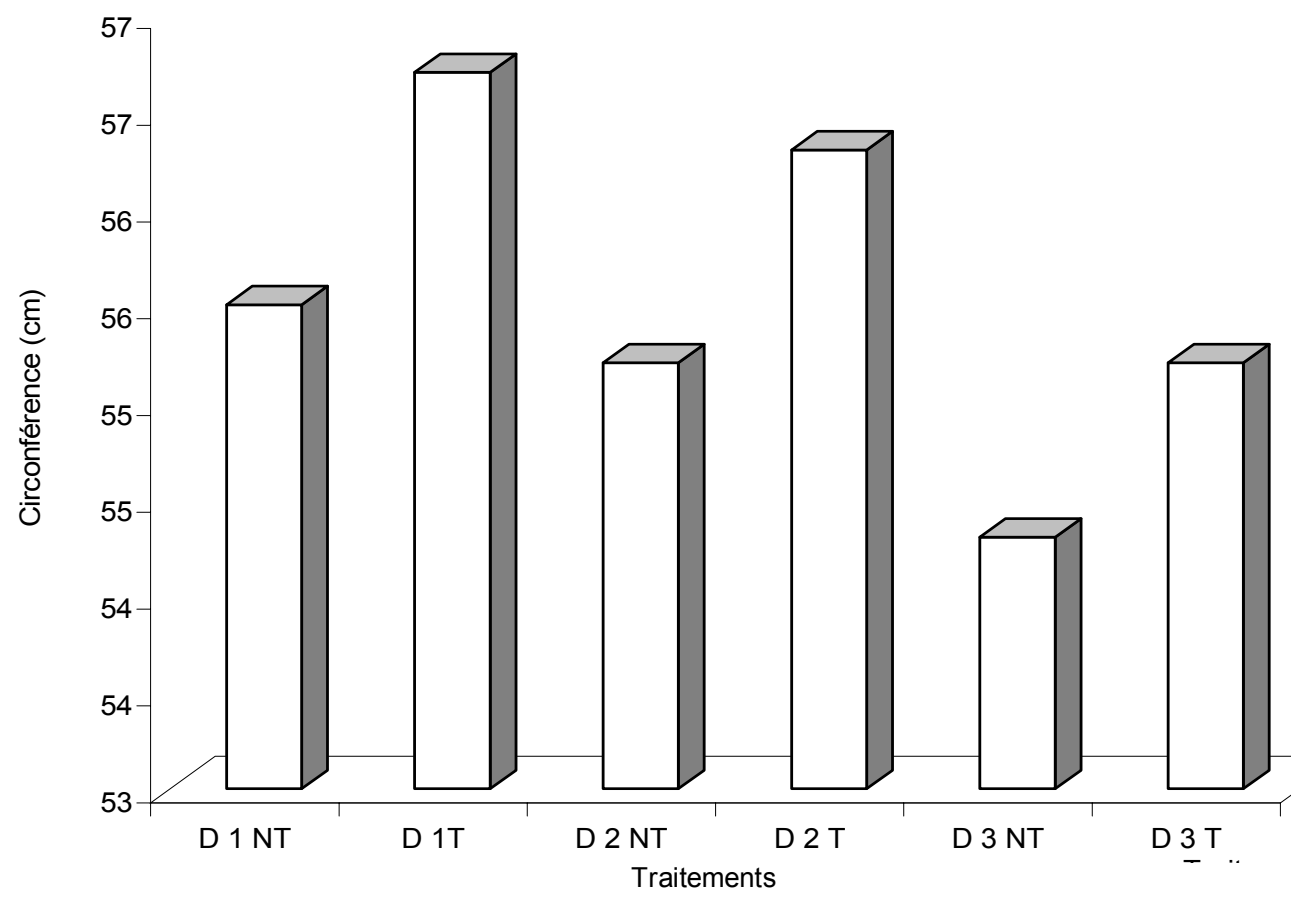

Figure 1 : Circonférence à la floraison du faux tronc des bananiers plantain traités $(T)$ et non traités (NT) avec du trifloxystrobine, à différentes densités de plantation.

Circumference at flowering of the plantain false trunk treated (T) and untreated (NT) with trifloxy strobin fungicide at different planting densities.

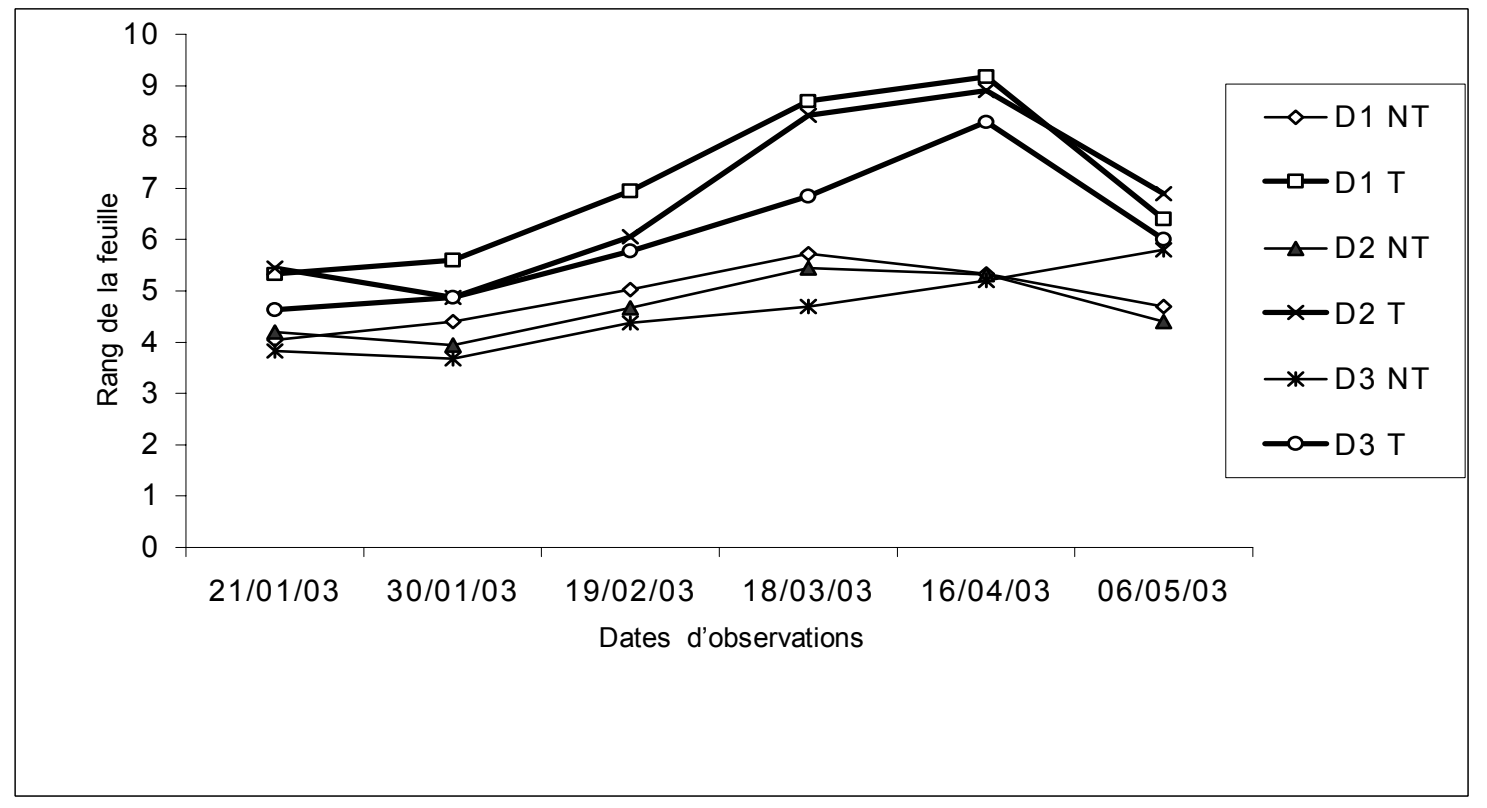

Figure 2 : Evolution de la plus jeune feuille avec tirets de MRN des bananiers plantain traités (T) et non (NT) avec du trifloxystrobine, à différentes densités de plantation.

Growth of the youngest leaf with black Sigatoka streaks of the plantain treated $(T)$ and untreated (NT) with trifloxystrobin fungicide at different planting densities. 


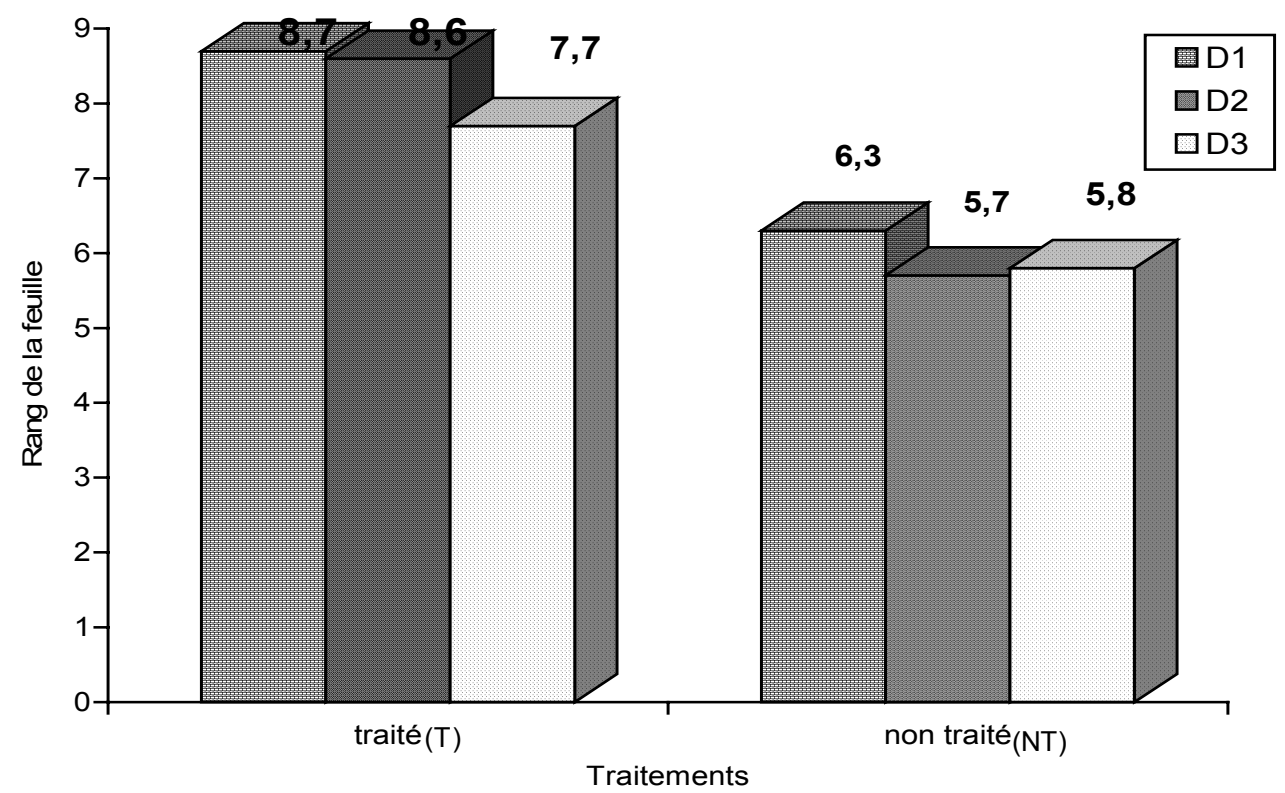

Figure 3 : Rang moyen de la plus jeune feuille nécrosée des bananiers plantain traités $(T)$ et non (NT) avec trifloxystrobine, à différentes densités de plantation.

Mean position of the youngest leaf with necrosis of black Sigatoka of the plantain treated (T) and untreated (NT) with trifloxystrobin fungicide at various densities of plantation.

Tableau 2 : Intervalle floraison-récolte et poids des régimes et des doigts en fonction des densités de plantation et du mode de gestion de la cercosporiose noire.

Flowering-harvest interval and bunches and fingers weight according to densities of cultivation and management mode of black Sigatoka.

\begin{tabular}{lcccccc}
\hline Traitements & D1 NT & D1 T & D2 NT & D2 T & D3 NT & D13T \\
\hline IFR & $74,86 \mathrm{~b}$ & $81,34 \mathrm{ab}$ & $76,16 \mathrm{~b}$ & $80,24 \mathrm{ab}$ & $79,31 \mathrm{ab}$ & 83,20 \\
PR & $13,98 \mathrm{ab}$ & $16,52 \mathrm{a}$ & $12,33 \mathrm{~b}$ & $13,24 \mathrm{~b}$ & $10,84 \mathrm{~b}$ & $11,85 \mathrm{~b}$ \\
PMD & $268,15 \mathrm{ab}$ & $288,50 \mathrm{a}$ & $253,81 \mathrm{ab}$ & $272,55 \mathrm{ab}$ & $237,27 \mathrm{~b}$ & $261,85 \mathrm{ab}$
\end{tabular}

IFR, Intervalle Floraison Récolte ; PR, Poids moyen du Régime ; Poids moyen du doigt

Par ligne, les moyennes suivies de la même lettre ne diffèrent pas statistiquement (test de Newman-Keuls au risque $\alpha=5 \%$ ).

\section{DISCUSSION}

Les résultats montrent que les applications du fongicide trifloxystrobine ont permis d'améliorer l'état végétatif et le rendement des bananiers plantain.

Concernant l'état végétatif, et quelle que soit la densité de plantation, le fongicide a permis d'accroître de façon significative le nombre de feuilles vivantes. Ce qui a entrainé une augmentation de la circonférence du pseudo tronc dans les parcelles traitées. L'évolution de la courbe de la PJFT a montré, pendant la phase précédant les applications du fongicide, des différences, traduisant un effet de densité de plantation. L'incidence de la maladie des raies noires a été d'autant plus sévère que la densité de plantation a été élevée. L'efficacité des traitements fongicides tardifs s'est traduite par une évolution ascendante des courbes jusqu'à un pallier, suivi d'une phase descendante (Figure 2). Cette situation résulte du fait que la phase phénologique correspond à la floraison des bananiers plantain. Ainsi, les feuilles sénescentes ne sont plus remplacées par de nouvelles émissions foliaires.

L'incidence des applications du fongicide sur l'état végétatif a varié avec la densité de plantation. Le nombre de feuilles vivantes, ainsi que les plus jeunes feuilles nécrosées ont été significativement les plus élevés pour la densité D2 (2500 plants/ha). Cette densité a été probablement la preuve d'une meilleure 
adéquation avec le brouillard fongicide reçu sur le feuillage. En effet, dans la mesure où le fongicide a été appliqué par rapport à la surface de la parcelle (non au nombre de plants traités), et au regard des densités, D1 a présenté une situation où le couvert végétal a été peu dense pour offrir une importante surface non couverte par le feuillage des bananiers plantain et par laquelle une bonne partie du fongicide parvient au sol et donc perdue. En revanche, D3 a offert une surface foliaire excessive par rapport au brouillard fongicide. II s'agit alors d'un effet de dilution du fongicide dans la biomasse foliaire. II eut été probablement plus intéressant d'augmenter proportionnellement la dose du fongicide et la quantité d'huile pour la densité D3.

En ce qui concerne le poids moyen du doigt et du régime, des augmentations significatives de 8 à $12 \%$, respectivement ont été observées par densité sur les parcelles traitées au fongicide. Si l'augmentation de la densité de plantation devait réduire l'incidence de la cercosporiose, ces différences n'auraient pas été significatives, surtout lorsque le programme d'application fongicide a été réduit au strict minimum. Or, concernant l'ensemble des données analysées, l'incidence de la cercosporiose noire s'est accrue avec la densité de plantation. L'étude, n'a pas permis de confirmer les résultats de Belalcázar et Cayon (2001). Selon ces auteurs, l'augmentation des densités de plantation créerait un microclimat dans lequel la température et l'humidité relative de l'air seraient stables. Ce qui défavoriserait l'explosion de la MRN. Ceci permettrait une gestion uniquement mécanique de la cercosporiose noire et affranchirait le producteur d'un recours aux fongicides. Notre étude, qui a porté sur des parcelles élémentaires de $300 \mathrm{~m}^{2}$, n'a pas permis d'atteindre cet objectif. L'atteinte d'un tel objectif nécessite la mise en œuvre de dispositions particulières comme l'amélioration de la fertilité des sols (amendements minéraux et organiques, drainage etc.) et surtout, l'augmentation de la taille des parcelles (au-delà d'un hectare).

\section{CONCLUSION}

La combinaison de la gestion mécanique de la MRN avec les applications de fongicide a permis de passer de 24 à seulement 4 applications annuelles. Les meilleurs résultats acquis jusques-là grâce à l'avertissement climatique, se sont situés entre 6 et 8 applications annuelles. Dans notre étude, 4 applications ont suffi jusqu'à la fin du cycle du bananier, avec environ $10 \%$ d'accroissement du rendement. Ce résultat serait meilleur avec 2 applications supplémentaires, c'est-à-dire avec 6 applications correspondant au plus bas niveau de traitement obtenu avec l'avertissement climatique. De plus, l'association de la forte densité de plantation avec le programme réduit de traitement chimique a amélioré aussi bien la productivité de la parcelle que le gain financier des planteurs en milieu rural.

\section{REMERCIEMENTS}

Les auteurs remercient I'INIBAP/IPGRI pour son appui financier à cette étude, et les Drs Ekow Akyeampong (coordinateur Musaco, INIBAP, au Cameroun) et Amoncho Adiko (CNRA, Côte d'Ivoire), pour leur contribution à l'obtention dudit financement.

\section{REFERENCES}

Belalcázar (S. C.) et (G. S.) Cayon. 2001. High density planting. INIBAP. LAC. 9p.

Burt (P. J. A.), (J.) Rutter et (H.) González. 1997. Short distance wind dispersal of the fungal pathogens causing Sigatoka diseases in banana and plantain. Plant pathology 46 (4) : 451-458.

Ganry (J.) et (J. P. ) Meyer . 1972a. La lutte contrôlée contre le cercospora aux Antilles. Bases climatiques de l'avertissement. Fruits 27 (10) : 665-676.

Ganry (J.) et (J. P.) Meyer. 1972b. La lutte contrôlée contre le cercospora aux Antilles. Techniques d'observation et de numération de la maladie. Fruits 27 (11) : 767-774.

Ganry (J.) et (E.) Laville. 1983. Les cercosporioses et leurs traitements. Evolution des méthodes de traitement : 1) traitements fongicides, 2) avertissement. Fruits, 38 (1) : 3-20.

Lassoudière (A.). 1978. Le bananier et sa culture en Côte d'Ivoire, première partie : connaissance de la plante, interaction avec le milieu écologique. Institut de Recherches sur les Fruits et Agrumes (IRFA), Abidjan, Côte d'Ivoire, 104 p. 